\title{
The association between fluid balance and mortality in patients with ARDS was modified by serum potassium levels: a retrospective study
}

Zhongheng Zhang, Lin Chen

Background and objective: acute respiratory distress syndrome (ARDS) is characterized by pulmonary edema and may benefit from conservative fluid management. However, conflicting results exist in the literature. The study aimed to investigate the association between mean fluid balance and mortality outcome in ARDS patients who required invasive mechanical ventilation. Methods: The study was a secondary analysis of a prospectively collected dataset obtained from the NHLBI Biologic Specimen and Data Repository Information Coordinating Center. ARDS patients with invasive mechanical ventilation were eligible. Demographic and laboratory data were extracted from the dataset. Multivariable regression model was built by stepwise selection of covariates. Fractional polynomial approach was used to test the linearity of mean fluid balance in the model. Potential interactions of mean fluid balance with other variables were tested. Main results: A total of 282 patients were eligible for the analysis, including 61 non-survivors with a mortality rate of $21.6 \%$. After stepwise regression analysis, mean fluid balance remained to be an independent predictor of death (OR: $1.00057 ; 95 \% \mathrm{Cl}$ : $1.00034-$ 1.00080). Two-term model obtained using fractional polynomial analysis was not superior to the linear model. There was significant interaction between mean fluid balance and serum potassium levels $(p=0.011)$. While the risk of death increased with increasing mean fluid balance at potassium levels of $1.9,2.9,3.9$ and $4.9 \mathrm{mmol} / \mathrm{l}$, the risk decreased at potassium level of $5.9 \mathrm{mmol} / \mathrm{l}$. Conclusion: The present study demonstrates that more positive fluid balance in the first 8 days is significantly associated with increased risk of death. However, the relationship between mean fluid balance and mortality can be modified by serum potassium levels. With hyperkalemia, more positive fluid balance is associated with reduced risk of death. 


\section{Title page}

2 The association between fluid balance 3 and mortality in patients with ARDS was 4 modified by serum potassium levels: a 5 retrospective study

6 Zhongheng ZHANG (MMed); Lin Chen (MMed)

7 Affiliation: Department of critical care medicine, Jinhua municipal central hospital, Jinhua

8 hospital of Zhejiang university, Zhejiang, P.R.China

9 Corresponding author: Zhongheng Zhang

10 Address: 351\#, Mingyue Road, Jinhua, Zhejiang province, China, 321000

11 Phone number: 86-579-82552667

12 Email: zh_zhang1984@hotmail.com

13 There are no conflicts of interest. 
Abstract

Background and objective: acute respiratory distress syndrome (ARDS) is characterized by pulmonary edema and may benefit from conservative fluid management. However, conflicting results exist in the literature. The study aimed to investigate the association between mean fluid balance and mortality outcome in ARDS patients who required invasive mechanical ventilation.

Methods: The study was a secondary analysis of a prospectively collected dataset obtained from the NHLBI Biologic Specimen and Data Repository Information Coordinating Center. ARDS patients with invasive mechanical ventilation were eligible. Demographic and laboratory data were extracted from the dataset. Multivariable regression model was built by stepwise selection of covariates. Fractional polynomial approach was used to test the linearity of mean fluid balance in the model. Potential interactions of mean fluid balance with other variables were tested.

Main results: A total of 282 patients were eligible for the analysis, including 61 nonsurvivors with a mortality rate of $21.6 \%$. After stepwise regression analysis, mean fluid balance remained to be an independent predictor of death (OR: $1.77 ; 95 \% \mathrm{Cl}: 1.42$ 2.22). Two-term model obtained using fractional polynomial analysis was not superior to the linear model. There was significant interaction between mean fluid balance and serum potassium levels $(p=0.011)$. While the risk of death increased with increasing mean fluid balance at potassium levels of 1.9, 2.9, 3.9 and $4.9 \mathrm{mmol} / \mathrm{l}$, the risk decreased at potassium level of $5.9 \mathrm{mmol} / \mathrm{l}$.

Conclusion: The present study demonstrates that more positive fluid balance in the first 8 days is significantly associated with increased risk of death. However, the relationship between mean fluid balance and mortality can be modified by serum potassium levels. With hyperkalemia, more positive fluid balance is associated with reduced risk of death. 
40 Acute respiratory distress syndrome (ARDS) is a leading cause of mortality and morbidity 41 for critically ill patients. The crude incidence of ARDS is reported to be around 80 per 42100,000 person-years, with in-hospital mortality rate of 38.5\%.(Rubenfeld et al. 2005) 43 However, these figures vary substantially due to different definitions of the syndrome. 44 ARDS is a clinical syndrome with several distinctive features: hypoxemia, pulmonary 45 infiltrates, proteinaceous pulmonary edema, acute onset after known insults, and 46 47 48 49 50 51 52 absence of elevated hydrostatic pressure as the cause. (Costa \& Amato 2013; Force et al. 2012) Great advances have been made in the management of ARDS in recent years (Roch et al. 2011), and ventilation strategy is the most extensively studied. Among various ventilation strategies, low-tidal volume ventilation, prone position, and high positive end-expiratory pressure (PEEP) have shown promising results. (Santa Cruz et al. 2011) Pharmacological treatment by using beta-agonist for the clearance of alveolar edema has also received attentions.(Ortiz-Diaz et al. 2013)

Because one of the most important pathological changes of ARDS is proteinaceous fluid accumulation in interstitial area of the lung, it follows that fluid restriction may be beneficial. Several investigations have been conducted to test this hypothesis. The results of these studies are conflicting. Fluid and Catheter Treatment (FACT) trial failed to demonstrate any beneficial effect on mortality outcome with conservative fluid administration, but it resulted in more days free of mechanical ventilation and ICU stay. (National Heart et al. 2006) On the other hand, some investigators demonstrated that conservative fluid management resulted in long term cognitive impairment.(Carlson \& Huang 2013; Mikkelsen et al. 2012) Most studies in the area utilized filling pressure to guide fluid management and direct daily fluid balance was not systematically analyzed with a rigorous multivariable model. The present study aimed to investigate the association between fluid balance and mortality outcome in ARDS patients by using rigorous model building strategy.

\section{Methods}

\section{Setting and Study population}

The study was a secondary analysis of data from a randomized controlled trial entitled "Randomized, Placebo-controlled Clinical Trial of an Aerosolized b2-Agonist for Treatment of Acute Lung Injury" (NCT 00434993).(National Heart et al. 2011) Dataset for this trial was obtained from National heart, lung, blood institute (NHLBI) Biologic Specimen and Data Repository Information Coordinating Center (https://biolincc.nhlbi.nih.gov/home/). The study was approved by the ethics committee of Jinhua municipal central hospital (2013-006) and informed consent was obtained in the original clinical trial.

Subjects of the study were recruited from 33 hospitals of the NHLBI ARDS Clinical Trial Network from August 6, 2007 to July 7, 2008. Patients were deemed eligible if 1) they had bilateral pulmonary infiltrates consistent with edema on chest X-ray; 2 ) had a ratio of arterial oxygen pressure (PaO2) to oxygen supply (FiO2) of $300 \mathrm{mmHg}$ or less; 3 ) had no clinical evidence of left atrial hypertension; and 4) they were intubated and mechanically ventilated. Exclusion criteria were patients with chronic lung disease, unable to obtain informed consent, time window exceeded, acute myocardial infarction, high 6 month mortality, chronic liver disease, physician refusal, not committed to full support, neuromuscular disease and other unknown reasons (see supplemental material of the original study for more details).(National Heart et al. 2011) Of the 2688 subjects being screened, 2406 were excluded due to these reasons. A total of 282 mechanically ventilated patients with ARDS were finally included in the dataset.

The dataset contained following information: demographics, types of ICU, causes of ARDS, comorbidity score (we assigned one point score for each additional one coexisting disease), lowest mean arterial pressure on admission, laboratory findings on enrollment 
90 (sodium, potassium, hemoglobin, glucose, bicarbonate, $\mathrm{PaO} 2 / \mathrm{FiO} 2, \mathrm{pH}$ value and serum 91 creatinine). Urine output, fluid intake and output were reported daily for up to 8 days or 92 until the subject left the study. The dataset recorded comorbidities including chronic 93 dialysis, leukemia, non-Hodgkin's Iymphoma, solid tumor with metastasis, immune 94 suppression, hepatic failure with coma or encephalopathy, cirrhosis, diabetes mellitus, 95 hypertension, prior myocardial infarction, congestive heart failure, peripheral vascular 96 disease, prior stroke with sequelae, dementia, chronic pulmonary disease, arthritis, 97 peptic ulcer disease.

98 Patients were followed up for 90 days and those who survived to 90 days were 99 considered as survivors. Otherwise, they were considered as non-survivors. Secondary outcome was unassisted breathing (UAB), which was defined as time to the first UAB. Subsequent return to mechanical ventilation was not taken into consideration.

102 Statistical analysis

103 Distribution of continuous variables was determined by inspection of the histogram and 104 tested based on skewness and kurtosis. Continuous data were expressed as mean and 105 standard deviation and median and interquartile range as appropriate, and their 106 comparisons were made by using t test or Wilcoxon rank-sum test. Categorical data 107 such as the types of ICU, causes of ARDS were expressed as the number and 108 percentage, and their differences between survivors and non-survivors were tested by 109 using Pearson's $\chi^{2}$ test.

110 Mortality was used as a binary outcome and logistic regression model was built to adjust 111 for potential confounders. The initial model included all variables with $p<0.3$ in bivariate 112 analysis (table 1). The main effect model was then established by using stepwise 113 backward elimination approach with the significance levels of removal and addition of 1140.2 and 0.1 , respectively. An important assumption for the continuous variable (mean 115 fluid balance) in Logistic regression model is that the variable is in linear relationship 116 with outcome in logit scale. If this assumption does not hold true, the fitted model may 117 not reflect the true relationship between mean fluid balance and mortality. We used 118 fractional polynomials to determine whether curvilinear model was better than the linear 119 model.(Royston P. 1994) We first determined the best fitting one-term and two-term 120 models by choosing power transformations from the set $\langle-2,-1,-0.5,0,0.5,1,2,3\rangle$, 121 where 0 denoted the log transformation. Then best fitting two-term model was 122 compared with linear model. If two-term model was significantly better than the linear 123 one $(p<0.05)$, two-term model was then compared to the best fitting one-term model. 124 Otherwise, linear model was adopted. The procedure proceeded until there was no 125 statistical significance and the best fitting model was chosen. This was termed the 126 closed test procedure.(W. Sauerbreia 2006) Interaction terms between mean fluid 127 balance and other variables in the main effect model were tested and terms with 128 statistical significance $(p<0.05)$ were included in the model.

129 Hosmer-Lemeshow goodness-of-fit test was performed to examine model fit. Another 130 important characteristic of fitted model is its discrimination power; that is, how accurate 131 can the fitted model predict outcome. We examined this by graphics. The probability of 132 death was depicted on the horizontal axis against the observed outcome in the $y$-axis. 133 Furthermore, the prediction power was assessed by using receiver operating 134 characteristics curve (ROC), and the area under the ROC was reported.(Royston \& 135 Altman 2010)

136 All statistical analyses was performed by using Stata 13 (StataCorp, College Station, 137 Texas 77845 USA), and a $p<0.05$ was considered to be statistically significant.

138 Results 
140 The baseline characteristics of included ARDS patients are shown in table 1 . There were

14161 non-survivors during the observation period with the mortality rate of $21.6 \%$. As

142 expected, non-survivors were significantly older than survivors (59.8 \pm 13.7 vs $49.4 \pm 16.2$

143 years, $p<0.001)$. There was no difference between survivors and non-survivors in

144 gender, ICU types, causes of ARDS, and the lowest mean blood pressure. However, there

145 were more comorbidity burden in non-survivors than in survivors (2.25 vs 1.40;

$146 \mathrm{p}<0.001)$. With respect to laboratory measurements on entry, only potassium and

147 bicarbonate were found to be associated with mortality outcome. Other variables were

148 not statistically significant. Survivors showed lower serum potassium levels than non-

149 survivors (3.90 \pm 0.58 vs $4.18 \pm 0.71 \mathrm{mEq} / \mathrm{l} ; \mathrm{p}=0.0019)$. $24 \mathrm{~h}$ urine output on day 0 was

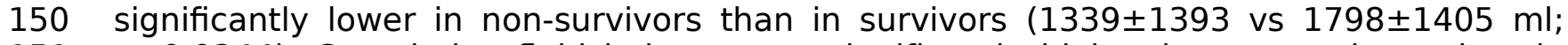

$151 \mathrm{p}=0.0244)$. Cumulative fluid balance was significantly higher in non-survivors than in

152 survivors ( $11614 \pm 13485$ vs $3578 \pm 9465 \mathrm{ml} ; \mathrm{p}<0.001)$; and mean fluid balance was also

153 significantly higher in non-survivors than in survivors (1913 2271 vs $427 \pm 1179 \mathrm{ml}$;

$154 \mathrm{p}<0.001$ ). Because there were 26 comparisons between survivors and non-survivors, it

155 was subject to the problem of multiple comparisons. A Bonferroni-adjusted significance

156 level of 0.002 was calculated to account for the increased possibility of type-l error. The

157 mean fluid balance remained statistically significant at this Bonferroni-adjusted

158 significance level. Figure 1 displays the distributions of fluid intake and output from day

1590 to day 8. More positive fluid balance was shown in the first 3 days, and thereafter the

160 fluid balance was approximately zero. The results of principal component analysis were

161 shown in figure 2. Two components were chosen because eigenvalues for the first two

162 principal component (PC) were greater than 1. Biplot shows the multi-dimensional data

163 were represented by two PCs. Biplot is a visualization technique for investigating the

164 inter-relationships between the observations and variables in multivariate data. The

165 component loading plot showed that PC loadings measure the importance of each

166 variable in accounting for the variability in the PC. PC scores are the derived composite

167 scores computed for each observation based on the eigenvectors for each PC.

168 After stepwise selection and elimination, five variables remained in the model (main 169 effect model, table 2): mean fluid balance (OR: $1.77 ; 95 \% \mathrm{Cl}: 1.42-2.22$ ), age (OR: 1.03 ;

170 95\% Cl: 1.01-1.06); potassium (OR: 1.84; 95\% Cl: 1.10-3.06); hemoglobin (OR: 0.83; 95\%

$171 \mathrm{Cl}$ : 0.68-1.00); and comorbidity (OR: 1.26; 95\% Cl: 0.97-1.64). In fractional polynomial

172 analysis, two-term model was significantly better than the model without the variable

173 (mean fluid balance) with a difference of deviance of $34.8(p<0.001)$. However, the two-

174 term model was not significantly better than the linear model (difference of deviance:

175 2.5; $p=0.111$ ), and thus the linear model was adopted for simplicity (table 3). When

176 interaction terms were entered into the model, we found that the term mean fluid

177 balancexpotassium was statistically significant, indicating that the effect of fluid

178 balance on mortality was modified by potassium levels. To make the result more

179 comprehensible to subject matter audience, graphical presentation of the result was

180 made in five potassium levels (figure 3). In all four levels, probability of death increased

181 exponentially with increasing mean fluid balance. Interestingly, in patients with

182 hyperkalemia, the probability of death decreased with increasing mean fluid balance.

183 This final model was well fitted as reflected by a Hosmer-Lemeshow $\chi 2$ of 5.26

$184(p=0.7292)$. Model discrimination was graphically shown in figure 4 . The scatter plot

185 showed that survivors were mostly gathered at the region with lower probability of

186 death, indicating a good negative predictive value of the model. The ROC curve showed

187 that the diagnostic performance of the model was excellent with and area under ROC of

188 0.84. Figure 5 displays Kaplan-Meier survivor and failure curves, stratified by median

189 mean fluid balance. The upper panel shows the probability of survival and the result

190 indicates that less mean fluid balance is associated with higher survival rate $(p=0.0007$

191 by rog-rank test). In the lower panel, less fluid balance is associated with higher rate of

192 returning to UAB ( $p<0.001$ with log-rank test). The results were robust after adjustment 
193 with other confounding factors in Cox proportional hazards model (table 5 and table 6).

194 Discussion

195 The present study demonstrates that mean fluid balance in the first 8 days is 196 significantly associated with mortality outcome. More positive fluid balance is associated 197 with significantly increased risk of death, and the result is robust after adjustment for 198 shock status, age, comorbidity and plasma hemoglobin. Unexpectedly, the relationship 199 between mean fluid balance and mortality can be modified by serum potassium levels. 200 With hyperkalemia, more positive fluid balance is associated with reduced risk of death. 201 Our study supports previous finding that conservative fluid management would be 202 beneficial for ARDS patients in a short term.

203

204

205

206

207

208

209

210

211

212

213

214

215

216

217

218

219

220

221

222

223

224

225

226

227

The first investigation into the fluid management in ARDS patients was conducted by Humphrey $\mathrm{H}$ and coworkers two decades ago.(Humphrey et al. 1990) However, they did not directly used fluid restriction strategy as the intervention, but divided patients into low and high pulmonary capillary wedge pressure (PCWP) groups. Although PCWP is correlated well with volume status, they are not good indicator of fluid responsiveness and can be influenced by multiple confounders. Therefore, the low PCWP may not well represent the optimized volume status, and it is impossible from this study to determine what is the quantity of fluid balance should be prescribed to achieve an optimal outcome. Mitchell JP and colleagues randomized patients with pulmonary artery catheter (PAC) in to PCWP-guided and EVLW-guided groups.(Mitchell et al. 1992) They found that EVLW-guided therapy was associated more negative fluid balance and favorable clinical outcomes. However, this study included heterogeneous patients with PAC, and was not specifically designed to explore the effect of fluid therapy in ARDS patients. The milestone study to investigate the effect of fluid restriction on acute lung injury (ALI) was the FACT trial,(National Heart et al. 2006) which was a multi-center randomized controlled trial enrolled 1000 patients with ALI. Conservative and liberal strategies were based on central venous pressure. The conservative group resulted in zero fluid balance in 7 days, whereas the liberal group resulted in +6 liter cumulative fluid balance. The conservative strategy improved oxygenation and appeared to reduce ICU length of stay, but had no beneficial effect on 60-day mortality. The major difference between our study and FACT trial is the severity of illness (ARDS requiring invasive mechanical ventilation vS ALI). Most probably, beneficial effect of fluid restriction can only be detected in ARDS patients in whom the pulmonary edema is more pronounced. In less severe form of lung injury such as ALI, the adverse effect of fluid restriction including tissue hypoperfusion and renal injury is the predominant net effect.(Prowle et al. 2014)

An interesting finding in our study was the interaction between serum potassium level and mean fluid balance, that is, the effect of mean fluid balance on mortality was modified by serum potassium level. In patients with hyperkalemia, mean fluid balance was negatively correlated with the probability of death (figure 2). The major cause of hyperkalemia in ICU patients was acute renal injury, and both hyperkalemia and acute renal injury were independent predictors of adverse outcome in critically ill patients. (Chertow et al. 2005; Goyal et al. 2012; Herrera-Gutierrez et al. 2013; Lassnigg et al. 2004) Initially, we postulated that the interaction effect was mediated by the kidney. In patients with high risk of renal injury or those with mild elevation in serum creatinine, electrolyte handling by the kidney is impaired and hyperkalemia may be induced. The progression of renal failure can be reversed with fluid resuscitation (more positive fluid balance) and ensuing improvement in renal perfusion. As a result, more positive fluid balance in patients with hyperkalemia appears to be beneficial. However, there is no sign that serum creatinine was elevated in the non-survivor group in our data and this postulation remains to be examined. In a retrospective study, McMahon GM and coworkers showed that potassium concentration was a strong independent predictor of 244 all-cause mortality within 30 days after ICU entry, and the effect persisted for one year. 
(McMahon et al. 2012) It is still unknown whether this association is causal and whether

246 strategies to reduce serum potassium level will improve outcome. Based on current 247 evidence, we proposed that more fluid intake would dilute potassium concentration, and 248 this in turn translated into improved outcome. Nevertheless, our study is hypothesis249 generating at this point, and further investigations are needed to verify this interesting 250 finding.

251 252 253 254

259

260

261

262

263

264

265

266

267

268

269

270

271

272

273

274

275

276

277

278

279

280

281

282

283

284

We employed rigorous methodology in model building and model check. An important concern in our study is that the relationship between mean fluid balance and mortality outcome may not be linear in logit scale. In study design period, we assumed that there would be one critical point above which more positive balance was harmful and below this point more positive balance was beneficial. This is a universal phenomenon that biological system often tries to keep variables within a normal range. For instance, our previous study demonstrated that ionized calcium was in U-shaped relationship with probability of death in critically ill.(Zhang et al. 2014b) However, the study failed to identify that fractional polynomial model was significantly better than the linear model and thus we can say from our analysis that less fluid balance is better for ARDS patients in the range of -2.5 to 10 liters per day. The study was limited by its observational nature because it was a secondary analysis of prospectively collected dataset. As described above, only $10 \%$ of ARDS patients were included for analysis and this small proportion may not be representative of the whole ARDS population. The majority of exclusion $(28.6 \%)$ was of unknown reason or not reported by participating centers. Of course, it is common in randomized controlled trials that only small proportion of patients fulfilled the inclusion/exclusion criteria.(Zhang et al. 2014a)

The study is limited by its small sample size. The established model may be unstable in future samples and the problem of overfitting may exist (Zhang 2014b). As a result, our study is hypothesis generating that requires further confirmation with larger sample size. One solution to this problem is to use clinical database that is established by using electronic medical record system (Zhang 2014a). Such a big data is characterized by large sample size and can support more degree of freedom in model building. Furthermore, the problem of multiple comparisons exists in our analysis, which may result in inflated type I error. To address this limitation, we employed Bonferroni-adjusted significance level to account for the increased possibility of type-I error. At this conservative significance level, the mean fluid balance remained statistically significant.

\section{Acknowledgement}

This Manuscript was prepared using ALTA Research Materials obtained from the NHLBI Biologic Specimen and Data Repository Information Coordinating Center and does not necessarily reflect the opinions or views of the ALTA or the NHLBI. 
Reference

286 Carlson CG, and Huang DT. 2013. The Adult Respiratory Distress Syndrome Cognitive Outcomes Study: long-term neuropsychological function in survivors of acute lung injury. Crit Care 17:317.

Chertow GM, Burdick E, Honour M, Bonventre JV, and Bates DW. 2005. Acute kidney injury, mortality, length of stay, and costs in hospitalized patients. J Am Soc Nephrol 16:3365-3370.

Costa EL, and Amato MB. 2013. The new definition for acute lung injury and acute respiratory distress syndrome: is there room for improvement? Curr Opin Crit Care 19:16-23.

Force ADT, Ranieri VM, Rubenfeld GD, Thompson BT, Ferguson ND, Caldwell E, Fan E, Camporota L, and Slutsky AS. 2012. Acute respiratory distress syndrome: the Berlin Definition. Jama 307:2526-2533.

Goyal A, Spertus JA, Gosch K, Venkitachalam L, Jones PG, Van den Berghe G, and Kosiborod M. 2012. Serum potassium levels and mortality in acute myocardial infarction. Jama 307:157-164.

Herrera-Gutierrez ME, Seller-Perez G, Sanchez-Izquierdo-Riera JA, Maynar-Moliner J, and group $\mathrm{Ci}$. 2013. Prevalence of acute kidney injury in intensive care units: the "COrte de prevalencia de disFuncion RenAl y DEpuracion en criticos" point-prevalence multicenter study. J Crit Care 28:687-694.

Humphrey H, Hall J, Sznajder I, Silverstein M, and Wood L. 1990. Improved survival in ARDS patients associated with a reduction in pulmonary capillary wedge pressure. Chest 97:1176-1180.

Lassnigg A, Schmidlin D, Mouhieddine M, Bachmann LM, Druml W, Bauer P, and Hiesmayr M. 2004. Minimal changes of serum creatinine predict prognosis in patients after cardiothoracic surgery: a prospective cohort study. J Am Soc Nephrol 15:1597-1605.

McMahon GM, Mendu ML, Gibbons FK, and Christopher KB. 2012. Association between hyperkalemia at critical care initiation and mortality. Intensive Care Med 38:18341842.

Mikkelsen ME, Christie JD, Lanken PN, Biester RC, Thompson BT, Bellamy SL, Localio AR, Demissie E, Hopkins RO, and Angus DC. 2012. The adult respiratory distress syndrome cognitive outcomes study: long-term neuropsychological function in survivors of acute lung injury. Am J Respir Crit Care Med 185:1307-1315.

Mitchell JP, Schuller D, Calandrino FS, and Schuster DP. 1992. Improved outcome based on fluid management in critically ill patients requiring pulmonary artery catheterization. Am Rev Respir Dis 145:990-998.

National Heart L, Blood Institute Acute Respiratory Distress Syndrome Clinical Trials $N$, Matthay MA, Brower RG, Carson S, Douglas IS, Eisner M, Hite D, Holets S, Kallet RH, Liu KD, Maclntyre N, Moss M, Schoenfeld D, Steingrub J, and Thompson BT. 2011. Randomized, placebo-controlled clinical trial of an aerosolized beta(2)-agonist for treatment of acute lung injury. Am J Respir Crit Care Med 184:561-568.

National Heart L, Blood Institute Acute Respiratory Distress Syndrome Clinical Trials N, Wiedemann HP, Wheeler AP, Bernard GR, Thompson BT, Hayden D, deBoisblanc B, Connors AF, Jr., Hite RD, and Harabin AL. 2006. Comparison of two fluid-management strategies in acute lung injury. N Engl J Med 354:2564-2575.

Ortiz-Diaz E, Festic E, Gajic O, and Levitt JE. 2013. Emerging pharmacological therapies for prevention and early treatment of acute lung injury. Semin Respir Crit Care Med 34:448-458.

Prowle JR, Kirwan CJ, and Bellomo R. 2014. Fluid management for the prevention and attenuation of acute kidney injury. Nat Rev Nephrol 10:37-47.

Roch A, Guervilly C, and Papazian L. 2011. Fluid management in acute lung injury and ards. Ann Intensive Care 1:16.

Royston P, and Altman DG. 2010. Visualizing and assessing discrimination in the logistic regression model. Stat Med 29:2508-2520.

Royston P. AD. 1994. Regression Using Fractional Polynomials of Continuous Covariates: Parsimonious Parametric Modelling. applied statistics 43:39.

Rubenfeld GD, Caldwell E, Peabody E, Weaver J, Martin DP, Neff M, Stern EJ, and Hudson LD. 2005. Incidence and outcomes of acute lung injury. N Engl J Med 353:1685-1693.

Santa Cruz R, Rojas JI, Nervi R, Heredia R, Ciapponi A, and Santa Cruz R. 2011. High versus 
low positive end-expiratory pressures (PEEP) levels for mechanically ventilated adult patients with acute lung injury and acute respiratory distress syndrome.

W. Sauerbreia CM-H, A. Bennerc, P. Roystond. 2006. Multivariable regression model building by using fractional polynomials: Description of SAS, STATA and R programs. computational statistics and data analysis 50:22.

Zhang Z. 2014a. Big data and clinical research: focusing on the area of critical care medicine in mainland China. Quant Imaging Med Surg 4:426-429.

Zhang Z. 2014b. Too much covariates in a multivariable model may cause the problem of overfitting. J Thorac Dis 6:E196-197.

Zhang Z, Ni H, and Xu X. 2014a. Observational studies using propensity score analysis underestimated the effect sizes in critical care medicine. J Clin Epidemiol 67:932-939.

Zhang Z, Xu X, Ni H, and Deng H. 2014b. Predictive value of ionized calcium in critically ill patients: an analysis of a large clinical database MIMIC II. PLOS ONE 9:e95204. 
356

357

358

359

360

361

362

363

364

365

366

367

368

369

370

371

372

373

374

375

376

377

378

379

380

381

382

383
Figure legends

Figure 1. Fluid intake and output from day 0 to day 8. More positive fluid balance was shown in the first 3 days, and thereafter the fluid balance was approximately zero.

Figure 2. Principal component analysis (PCA) for the multivariate dataset. Two components were chosen because eigenvalues for the first two principal component (PC) were greater than 1. Biplot shows the multi-dimensional data were represented by two PCs. Biplot (panel B) is a visualization technique for investigating the inter-relationships between the observations and variables in multivariate data. The component loading plot showed that PC loadings measure the importance of each variable in accounting for the variability in the PC. PC scores are the derived composite scores computed for each observation based on the eigenvectors for each PC.

Figure 3. Graphical presentation of the association between mean fluid balance and probability of death, stratified by serum potassium levels. "S"-shaped relationship between mean fluid balance and risk of mortality was shown for potassium levels at 1.9, 2.9 and $3.9 \mathrm{mmol} / \mathrm{l}$. The relationship was more linear at potassium level of $4.9 \mathrm{mmol} / \mathrm{l}$. Inverse relationship between mean fluid balance and risk of mortality was found at potassium level of $5.9 \mathrm{mmol} / \mathrm{l}$. The relationship was not sensitive to potassium levels (in panel $B$ we set potassium levels at 2, 3, 4, 5 and 6).

Figure 4. Graphical presentation of model discrimination. The scatter plot (A) showed that survivors were mostly gathered at the region with lower probability of death (left $\mathrm{x}$ axis), indicating a good negative predictive value of the model. The ROC curve (C) showed that the diagnostic performance of the model was excellent with and area under ROC of 0.84 .

Figure 5. Kaplan-Meier survivor and failure curves, stratified by median mean fluid balance. Panel A shows the probability of survival and the result indicates that less mean fluid balance is associated with higher survival rate ( $p=0.0007$ by rog-rank test). In panel $B$, less fluid balance is associated with higher rate of returning to UAB $(p<0.001$ with log-rank test). 


\section{Table $\mathbf{1}_{\text {(on next page) }}$}

Table 1 Demographics and baseline clinical characteristics of ARDS patients by survival status

If Others include trauma, coronary care unit, burn care unit, cardiac surgery ICU, and neuro ICU. † comorbidities include chronic dialysis, leukemia, non-Hodgkin's lymphoma, solid tumor with metastasis, immune suppression, hepatic failure with coma or encephalopathy, cirrhosis, diabetes mellitus, hypertension, prior myocardial infarction, congestive heart failure, peripheral vascular disease, prior stroke with sequelae, dementia, chronic pulmonary disease, arthritis, peptic ulcer disease. Abbreviations: ICU, intensive care unit; ARDS, acute respiratory distress syndrome; 


\section{PeerJ Reviewing Manuscript}

Table 1 Demographics and baseline clinical characteristics of ARDS patients by survival status

\begin{tabular}{|c|c|c|c|c|}
\hline Variables & $\begin{array}{l}\text { Overall } \\
(n=282)\end{array}$ & $\begin{array}{l}\text { Survivors } \\
(n=221)\end{array}$ & $\begin{array}{l}\text { Non-survivors } \\
(n=61)\end{array}$ & P value \\
\hline Age (years) & $51.6 \pm 16.2$ & $49.4 \pm 16.2$ & $59.8 \pm 13.7$ & $<0.001$ \\
\hline Male $(n, \%)$ & $156(55.32)$ & $120(54.30)$ & $36(59.02)$ & 0.512 \\
\hline \multicolumn{5}{|l|}{$\begin{array}{l}\text { ICU types (n, } \\
\%)\end{array}$} \\
\hline Medical ICU & $157(55.67)$ & $121(54.75)$ & $36(59.02)$ & 0.553 \\
\hline Mixed ICU & 54 (19.15) & $45(20.36)$ & $9(14.75)$ & 0.324 \\
\hline Surgical ICU & 31 (10.99) & $25(11.31)$ & $6(9.84)$ & 0.744 \\
\hline Others & $40(14.18)$ & $30(13.57)$ & $10(16.39)$ & 0.576 \\
\hline \multicolumn{5}{|l|}{$\begin{array}{l}\text { Causes of } \\
\text { ARDS }(n, \%)\end{array}$} \\
\hline Sepsis & $156(55.32)$ & $118(53.39)$ & $38(62.30)$ & 0.216 \\
\hline Transfusion & $14(4.96)$ & $12(5.43)$ & $2(3.28)$ & 0.494 \\
\hline Aspiration & $71(25.18)$ & $54(24.43)$ & $17(27.87)$ & 0.584 \\
\hline Pneumonia & $165(58.51)$ & $130(58.82)$ & $35(57.38)$ & 0.839 \\
\hline Others & $24(8.51)$ & $22(9.95)$ & $2(3.28)$ & 0.098 \\
\hline $\begin{array}{l}\text { Comorbidity } \\
\text { score } \dagger\end{array}$ & $1.59 \pm 1.34$ & $1.40 \pm 1.28$ & $2.25 \pm 1.35$ & $<0.001$ \\
\hline $\begin{array}{l}\text { Lowest mean } \\
\text { arterial } \\
\text { pressure } \\
(\mathrm{mmHg})\end{array}$ & $60.9 \pm 11.4$ & $61.5 \pm 11.9$ & $58.8 \pm 9.3$ & 0.1053 \\
\hline \multicolumn{5}{|l|}{$\begin{array}{l}\text { On } \\
\text { enrollment } \\
\text { laboratory }\end{array}$} \\
\hline $\begin{array}{l}\text { Hemoglobin } \\
(\mathrm{g} / \mathrm{dl})\end{array}$ & $10.40 \pm 2.22$ & $10.52 \pm 2.30$ & $9.95 \pm 1.82$ & 0.075 \\
\hline $\begin{array}{l}\text { Sodium } \\
(\mathrm{mmol} / \mathrm{l})\end{array}$ & $138.9 \pm 5.7$ & $138.9 \pm 5.6$ & $138.9 \pm 6.4$ & 0.997 \\
\hline $\begin{array}{l}\text { Potassium } \\
(\mathrm{mEq} / \mathrm{l})\end{array}$ & $3.96 \pm 0.62$ & $3.90 \pm 0.58$ & $4.18 \pm 0.71$ & 0.0019 \\
\hline $\begin{array}{l}\text { Glucose } \\
\text { (mg /dl) }\end{array}$ & $126.4 \pm 55.0$ & $128.1 \pm 50.4$ & $121.4 \pm 69.2$ & 0.402 \\
\hline $\begin{array}{l}\text { Bicarbonate } \\
(\mathrm{mEq} / \mathrm{l})\end{array}$ & $22.56 \pm 5.50$ & $23.03 \pm 5.49$ & $20.84 \pm 5.23$ & 0.0056 \\
\hline $\begin{array}{l}\mathrm{PaO} 2 / \mathrm{FiO} 2 \\
(\mathrm{mmHg})\end{array}$ & $\begin{array}{l}161.76 \pm 78.9 \\
0\end{array}$ & $\begin{array}{l}160.30 \pm 82.8 \\
2\end{array}$ & $\begin{array}{l}166.92 \pm 78.9 \\
0\end{array}$ & 0.5813 \\
\hline${ }_{(\mathrm{mmHg})}^{\mathrm{PaCO} 2}$ & $40.01 \pm 11.83$ & $40.20 \pm 10.82$ & $39.35 \pm 14.97$ & 0.6232 \\
\hline $\mathrm{pH}$ value & $7.35 \pm 0.10$ & $7.35 \pm 0.10$ & $7.31 \pm 0.11$ & 0.0075 \\
\hline $\begin{array}{l}\text { creatinine } \\
(\mathrm{mg} / \mathrm{dl})\end{array}$ & $1.91 \pm 1.65$ & $1.85 \pm 1.71$ & $2.10 \pm 1.43$ & 0.2946 \\
\hline \begin{tabular}{ll}
\multicolumn{3}{l}{ Urine } & output \\
day & 0 \\
(ml/24h)
\end{tabular} & $1698 \pm 1413$ & $1798 \pm 1405$ & $1339 \pm 1393$ & 0.0244 \\
\hline $\begin{array}{l}\text { Fluid balance } \\
\text { day } 0 \quad(\mathrm{ml} / 24 \\
\text { h) }\end{array}$ & $2814 \pm 3590$ & $2588 \pm 3435$ & $3628 \pm 4025$ & 0.045 \\
\hline $\begin{array}{l}\text { Cumulative } \\
\text { balance in } 8 \\
\text { days }\end{array}$ & $5317 \pm 10952$ & $3578 \pm 9465$ & $\begin{array}{l}11614 \pm 1348 \\
5\end{array}$ & $<0.001$ \\
\hline Mean fluid & $749 \pm 1601$ & $427 \pm 1179$ & $1913 \pm 2271$ & $<0.001$ \\
\hline
\end{tabular}




\section{PeerJ Reviewing Manuscript}

\begin{tabular}{|l|l|l|l|}
$\begin{array}{l}\text { balance in } 8 \\
\text { days }\end{array}$ & & & \\
\hline
\end{tabular}

ICU, and neuro ICU.

† comorbidities include chronic dialysis, leukemia, non-Hodgkin's lymphoma, solid tumor with metastasis, immune suppression, hepatic failure with coma or encephalopathy, cirrhosis, diabetes mellitus, hypertension, prior myocardial infarction, congestive heart failure, peripheral vascular disease, prior stroke with sequelae, dementia, chronic pulmonary disease, arthritis, peptic ulcer disease.

Abbreviations: ICU, intensive care unit; ARDS, acute respiratory distress syndrome; 


\section{Table 2 (on next page)}

Table 2 Main effect model after stepwise selection of covariates $†$

†In this multivariable model, mortality was treated as the binary dependent variable. The initial model was built by incorporating all variables with $p<0.3$ in bivariate analysis. The main effect model was established by using stepwise backward elimination approach with the significance levels of removal and addition of 0.2 and 0.1 , respectively. 


\section{PeerJ Reviewing Manuscript}

Table 2 Main effect model after stepwise selection of covariates†

\begin{tabular}{|l|l|l|l|l|l|}
\hline & Odds ratio & $\begin{array}{l}\text { Standard } \\
\text { error }\end{array}$ & $\begin{array}{l}\text { Lower limit } \\
\text { of 95\% Cl }\end{array}$ & $\begin{array}{l}\text { Upper limit } \\
\text { of 95\% Cl }\end{array}$ & $\mathrm{p}$ \\
\hline $\begin{array}{l}\text { Mean } \\
\text { balance } \\
\text { (with each } \\
\begin{array}{l}\text { 1000ml } \\
\text { increase) }\end{array}\end{array}$ & 1.77 & 0.20 & 1.42 & 2.22 & $<0.001$ \\
\hline Age & 1.03 & 0.01 & 1.01 & 1.06 & 0.01 \\
\hline $\begin{array}{l}\text { Comorbidit } \\
\text { y }\end{array}$ & 1.26 & 0.17 & 0.97 & 1.64 & 0.09 \\
\hline $\begin{array}{l}\text { Hemoglobi } \\
\mathrm{n}\end{array}$ & 0.83 & 0.08 & 0.68 & 1.00 & 0.05 \\
\hline Potassium & 1.84 & 0.48 & 1.10 & 3.06 & 0.02 \\
\hline
\end{tabular}

†In this multivariable model, mortality was treated as the binary dependent variable. The initial model was built by incorporating all variables with $p<0.3$ in bivariate analysis. The main effect model was established by using stepwise backward elimination approach with the significance levels of removal and addition of 0.2 and 0.1 , respectively. 


\section{Table 3 (on next page)}

Table 3 Comparisons of fractional polynomial models

Model selection was performed using closed test procedure. By comparing to the model without variable (omitted model), two-term model $(m=2)$ was significantly better with a difference of deviance of 34.8. However, two-term model was not significantly better than the linear model (difference of deviance: $2.5 ; p=0.111$ ), and thus the linear model was adopted for simplicity. 


\section{PeerJ Reviewing Manuscript}

Table 3 Comparisons of fractional polynomial models

\begin{tabular}{|c|c|c|c|c|c|}
\hline $\begin{array}{l}\text { Mean } \\
\text { balance }\end{array}$ & $\begin{array}{l}\text { Degree of } \\
\text { freedom }\end{array}$ & Deviance & $\begin{array}{l}\text { Difference } \\
\text { of } \\
\text { deviance }\end{array}$ & $p$ & Powers \\
\hline Omitted & 0 & 257.927 & 34.789 & 0.000 & \\
\hline Linear & 1 & 225.680 & 2.542 & 0.111 & 1 \\
\hline$m=1$ & 1 & 223.775 & 0.637 & 0.425 & 3 \\
\hline$m=2$ & 2 & 223.138 & 0.000 & -- & 3 \\
\hline
\end{tabular}

Model selection was performed using closed test procedure. By comparing to the model without variable (omitted model), two-term model $(m=2)$ was significantly better with a difference of deviance of 34.8. However, two-term model was not significantly better than the linear model (difference of deviance: $2.5 ; p=0.111$ ), and thus the linear model was adopted for simplicity. 


\section{Table 4(on next page)}

Table 4 Final model including interaction terms $†$

† All possible interactions between mean balance and other covariates were evaluated and only the term Mean balancexpotassium was statistically significant. Goodness-of-fit test showed the Hosmer-Lemeshow $\chi^{2}$ was $5.26(p=0.7292)$. 


\section{PeerJ Reviewing Manuscript}

Table 4 Final model including interaction terms $†$

\begin{tabular}{|l|l|l|l|}
\hline Variables & Odds ratio & $95 \% \mathrm{Cl}$ & P value \\
\hline Age & 1.03 & $1.007-1.057$ & 0.011 \\
\hline Potassium & 3.18 & $1.63-6.20$ & 0.001 \\
\hline Hemoglobin & 0.82 & $0.67-0.99$ & 0.043 \\
\hline $\begin{array}{l}\text { Comorbidity (0 as } \\
\text { the reference) }\end{array}$ & & & \\
\hline 1 & 2.22 & $0.69-7.15$ & 0.180 \\
\hline 2 & 1.38 & $0.40-4.83$ & 0.612 \\
\hline 3 & 3.88 & $1.16-13.0$ & 0.028 \\
\hline 4 & 2.96 & $0.60-14.6$ & 0.182 \\
\hline 5 & 0.82 & $0.09-7.54$ & 0.861 \\
\hline Mean balance & 1.003 & $1.001-1.004$ & 0.001 \\
\hline $\begin{array}{l}\text { Mean } \\
\text { balancexpotassiu } \\
\text { m }\end{array}$ & 0.9995 & $0.9991-0.9999$ & 0.011 \\
\hline
\end{tabular}

$\dagger$ All possible interactions between mean balance and other covariates were evaluated and only the term Mean balancexpotassium was statistically significant. Goodness-of-fit test showed the Hosmer-Lemeshow $\chi^{2}$ was 5.26 $(p=0.7292)$. 


\section{Table 5(on next page)}

Table 5 Cox proportional hazards model for mortality 


\section{PeerJ Reviewing Manuscript}

Table 5 Cox proportional hazards model for mortality

\begin{tabular}{|l|l|l|l|l|l|}
\hline & $\begin{array}{l}\text { Hazard } \\
\text { ratio }\end{array}$ & $\begin{array}{l}\text { Standard } \\
\text { error }\end{array}$ & $\begin{array}{l}\text { Lower limit } \\
\text { of 95\% Cl }\end{array}$ & $\begin{array}{l}\text { Upper limit } \\
\text { of 95\% Cl }\end{array}$ & $p$ \\
\hline $\begin{array}{l}\text { Mean } \\
\text { balance } \\
\text { (with each } \\
\begin{array}{l}\text { 1000ml } \\
\text { increase) }\end{array}\end{array}$ & 1.58 & 0.10 & 1.40 & 1.79 & 0.00 \\
\hline Age & 1.02 & 0.01 & 1.00 & 1.03 & 0.05 \\
\hline $\begin{array}{l}\text { Comorbidit } \\
\text { y }\end{array}$ & 1.30 & 0.13 & 1.06 & 1.59 & 0.01 \\
\hline $\begin{array}{l}\text { Hemoglobi } \\
n\end{array}$ & 0.92 & 0.07 & 0.80 & 1.06 & 0.24 \\
\hline Potassium & 1.46 & 0.24 & 1.06 & 2.00 & 0.02 \\
\hline
\end{tabular}


Table 6(on next page)

Table 6 Cox proportional hazards model for unassisted breathing 


\section{PeerJ Reviewing Manuscript}

Table 6 Cox proportional hazards model for unassisted breathing

\begin{tabular}{|l|l|l|l|l|l|}
\hline & $\begin{array}{l}\text { Hazard } \\
\text { ratio }\end{array}$ & $\begin{array}{l}\text { Standard } \\
\text { error }\end{array}$ & $\begin{array}{l}\text { Lower limit } \\
\text { of 95\% Cl }\end{array}$ & $\begin{array}{l}\text { Upper limit } \\
\text { of 95\% Cl }\end{array}$ & $p$ \\
\hline $\begin{array}{l}\text { Mean } \\
\text { balance } \\
\text { (with each } \\
\begin{array}{l}\text { 1000ml } \\
\text { increase) }\end{array}\end{array}$ & 0.71 & 0.04 & 0.64 & 0.79 & 0.00 \\
\hline Age & 1.00 & 0.00 & 0.99 & 1.01 & 0.98 \\
\hline $\begin{array}{l}\text { Comorbidit } \\
\text { y }\end{array}$ & 0.91 & 0.05 & 0.81 & 1.02 & 0.11 \\
\hline $\begin{array}{l}\text { Hemoglobi } \\
n\end{array}$ & 1.00 & 0.03 & 0.94 & 1.06 & 0.92 \\
\hline Potassium & 0.83 & 0.09 & 0.67 & 1.03 & 0.09 \\
\hline
\end{tabular}


1

Fluid intake and output from day 0 to day 8.

More positive fluid balance was shown in the first 3 days, and thereafter the fluid balance was approximately zero.

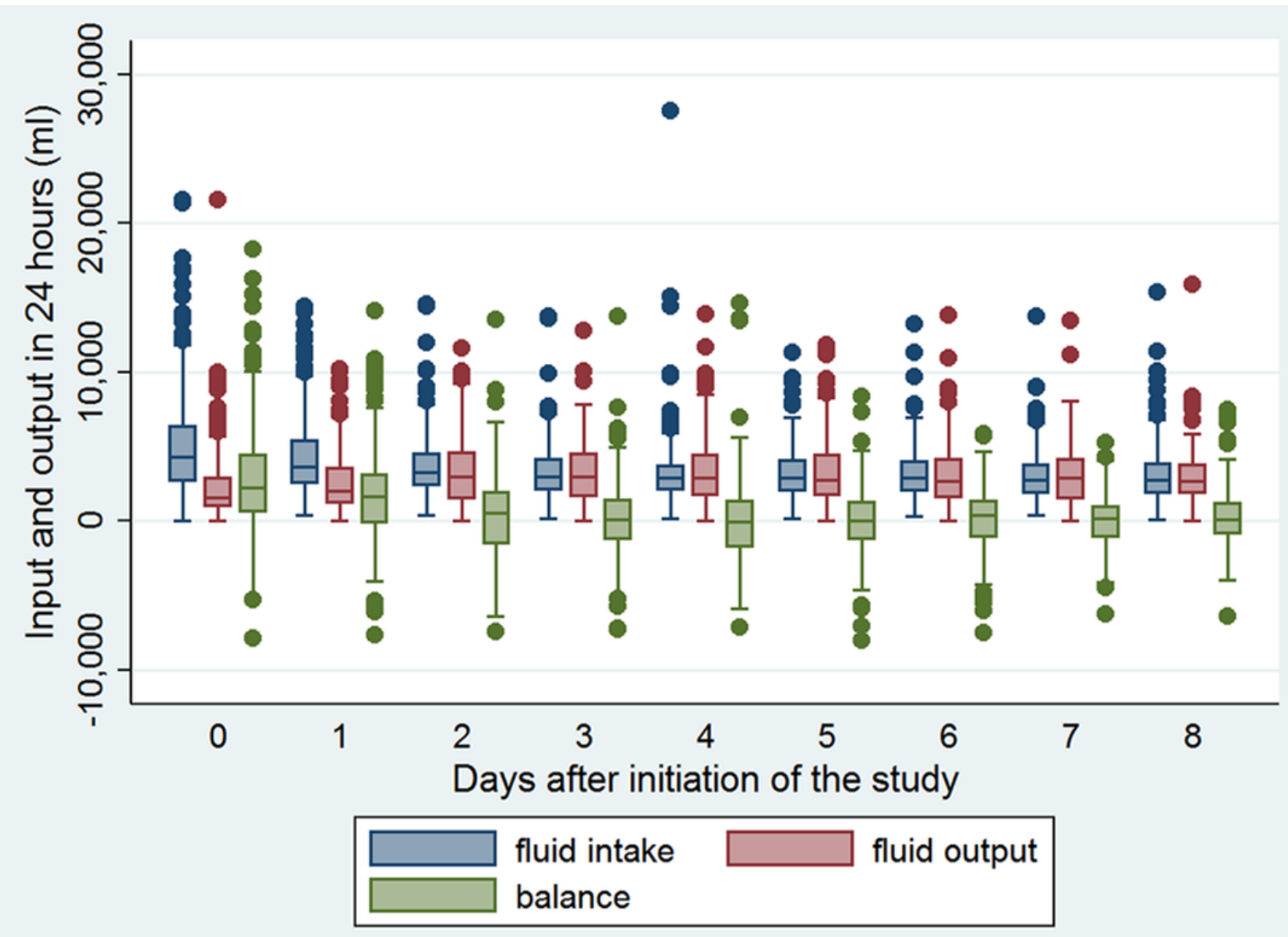


Principal component analysis (PCA) for the multivariate dataset.

Two components were chosen because eigenvalues for the first two principal component (PC) were greater than 1. Biplot shows the multi-dimensional data were represented by two PCs. Biplot (panel B) is a visualization technique for investigating the inter-relationships between the observations and variables in multivariate data. The component loading plot showed that PC loadings measure the importance of each variable in accounting for the variability in the PC. PC scores are the derived composite scores computed for each observation based on the eigenvectors for each PC.

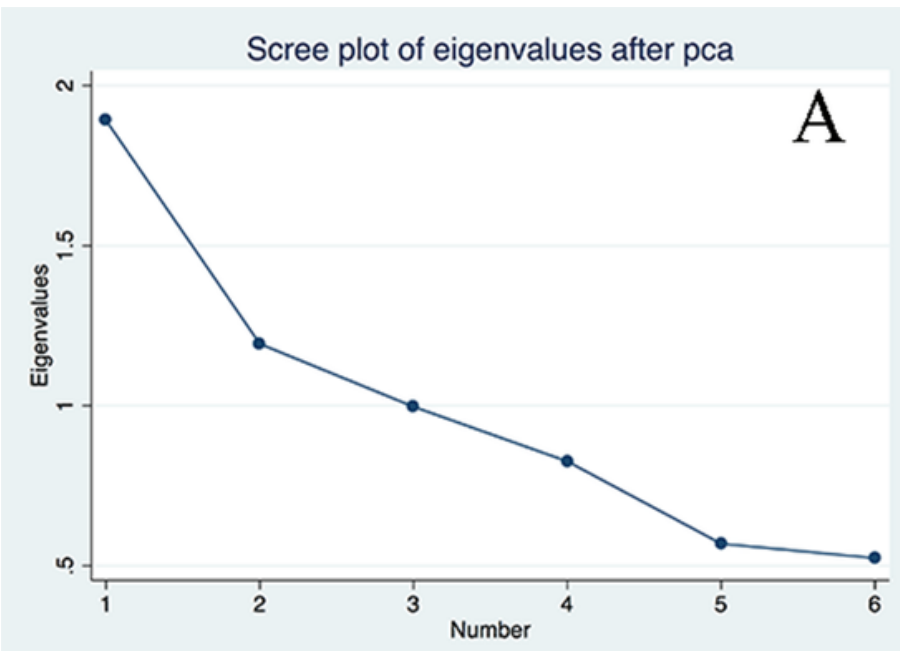

Component loadings

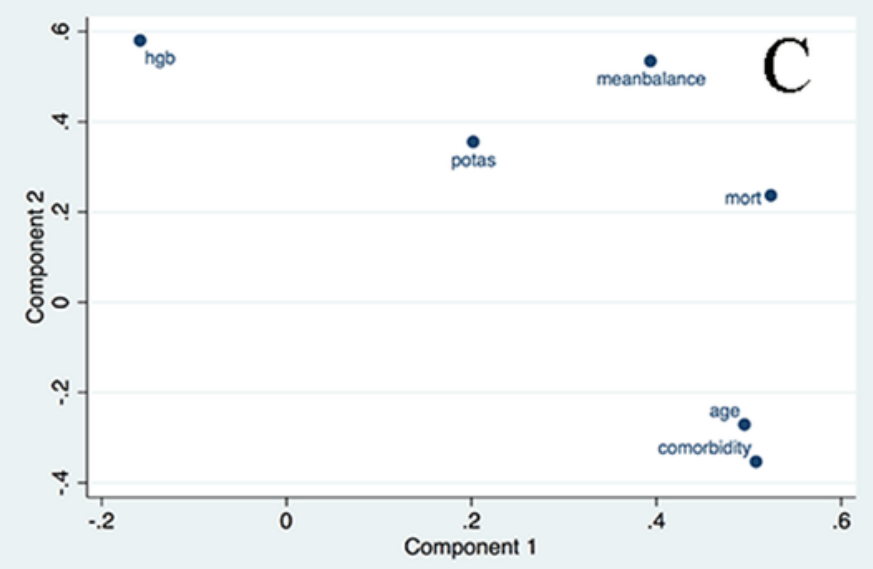

Biplot

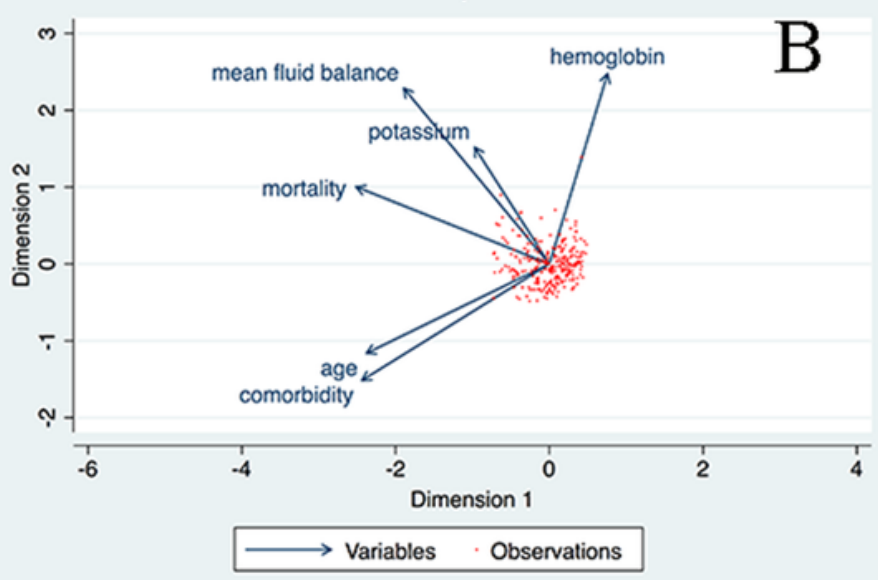

Score variables (pca)

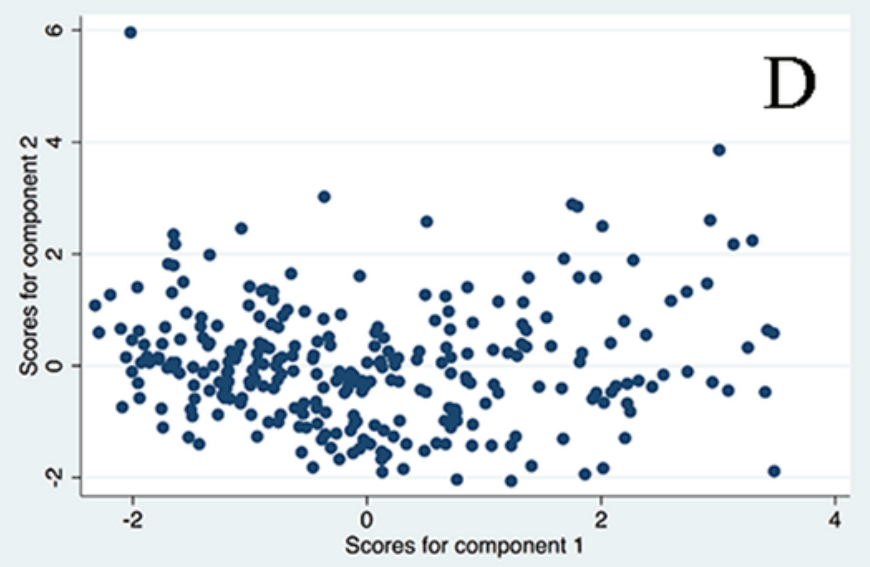




\section{3}

Graphical presentation of the association between mean fluid balance and probability of death, stratified by serum potassium levels.

"S"-shaped relationship between mean fluid balance and risk of mortality was shown for potassium levels at 1.9, 2.9 and $3.9 \mathrm{mmol} / \mathrm{l}$. The relationship was more linear at potassium level of $4.9 \mathrm{mmol} / \mathrm{l}$. Inverse relationship between mean fluid balance and risk of mortality was found at potassium level of $5.9 \mathrm{mmol} / \mathrm{l}$. The relationship was not sensitive to potassium levels (in panel B we set potassium levels at 2, 3, 4, 5 and 6). 

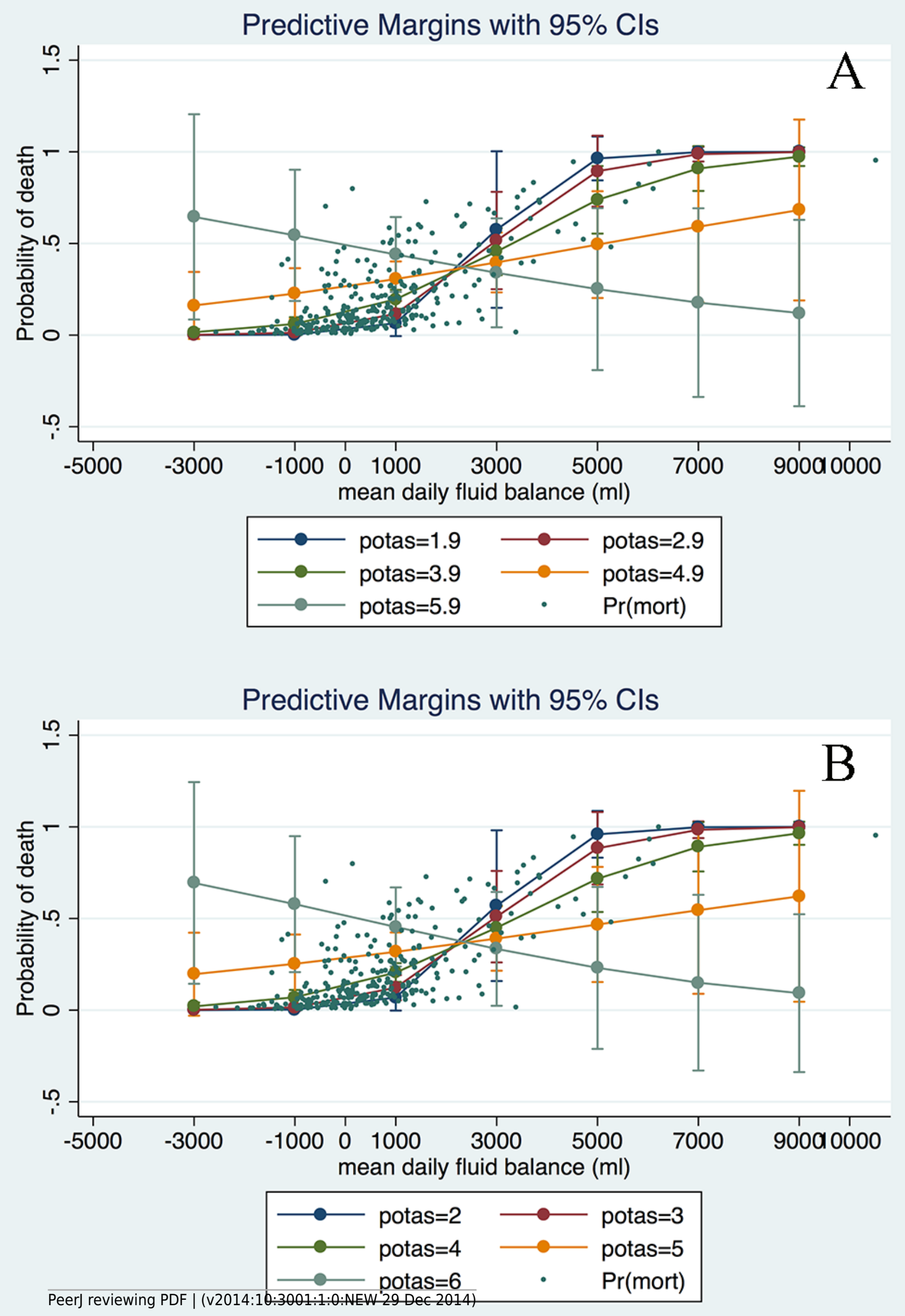
4

Graphical presentation of model discrimination.

The scatter plot (A) showed that survivors were mostly gathered at the region with lower probability of death (left x-axis), indicating a good negative predictive value of the model. The ROC curve $(C)$ showed that the diagnostic performance of the model was excellent with and area under ROC of 0.84 .
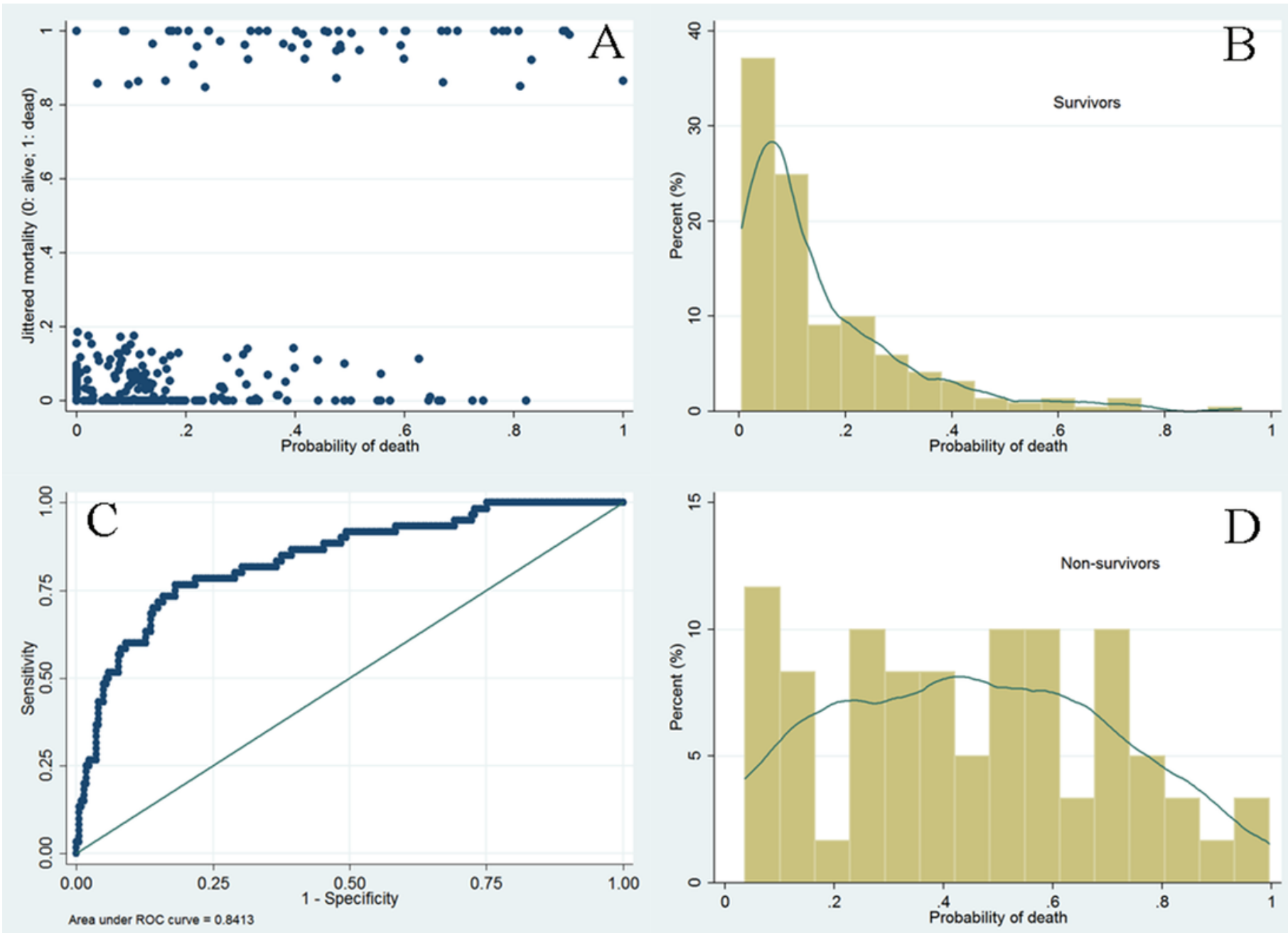


\section{5}

Kaplan-Meier survivor and failure curves, stratified by median mean fluid balance.

Panel A shows the probability of survival and the result indicates that less mean fluid balance is associated with higher survival rate ( $p=0.0007$ by rog-rank test). In panel $B$, less fluid balance is associated with higher rate of returning to $\operatorname{UAB}(p<0.001$ with log-rank test). 

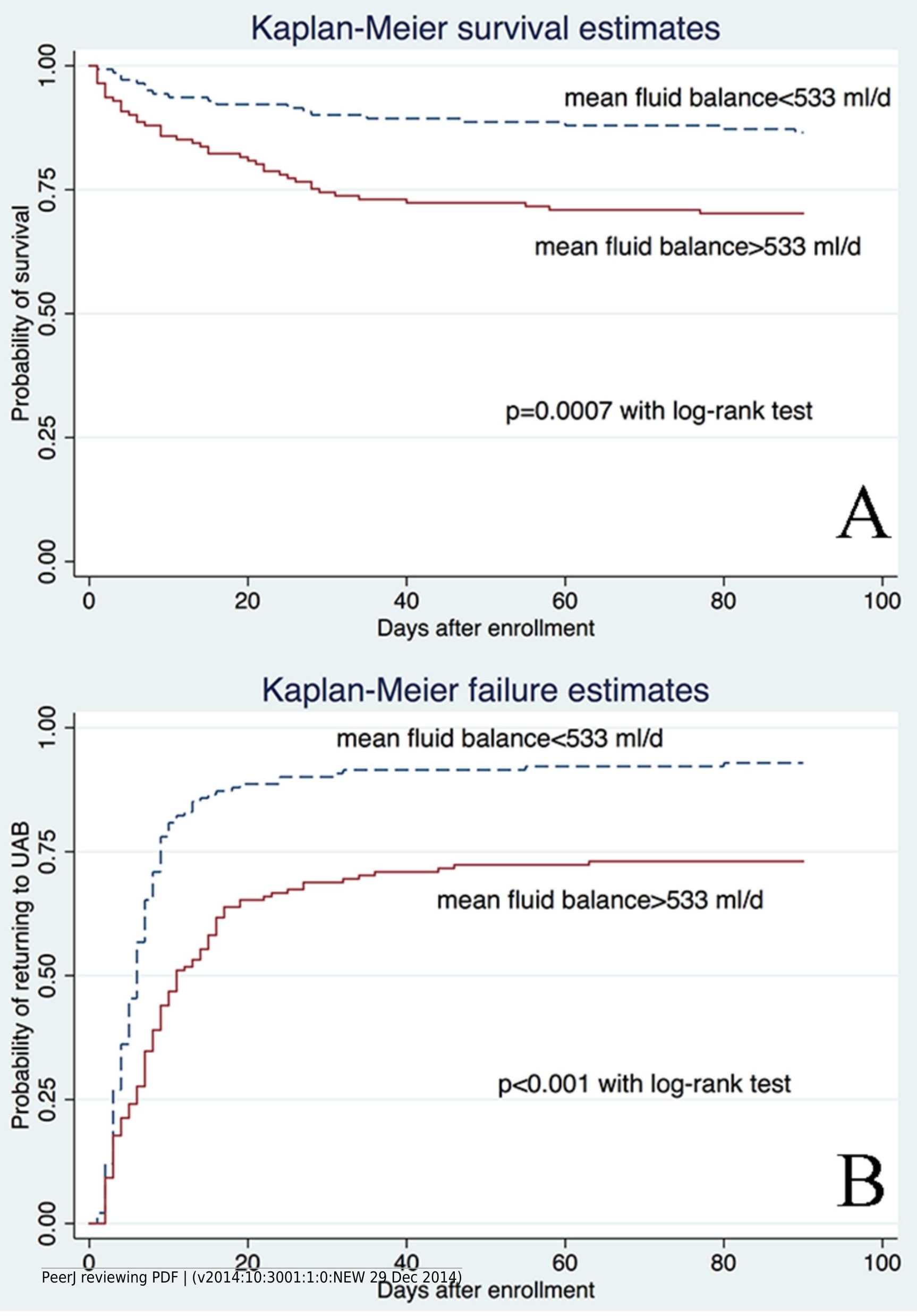